\title{
LETTER
}

\section{Topical nystatin prophylaxis: a likely viable measure for reducing impact of candidemia in neurosurgical patients with early tracheostomy intubation}

\author{
Giancarlo Ceccarelli,**, Simone Giuliano', Marco Falcone² and Mario Venditti ${ }^{1}$ \\ See related research by Giglio et al., http://ccforum.com/content/16/2/R57
}

Only a few reports have been published on the use of topical antifungal prophylaxis [1-4]. For this reason we read with interest the paper published by Giglio and colleagues [5] underlining the ability of oral nystatin to reduce fungal colonization in surgical/trauma ICU patients. In our experience, we observed a dramatic increase of candidemia prevalence in neurosurgical ICU patients after implementation of early tracheostomy (from 2.2\% to 9.7\%). Following an analysis of data collected, we developed the idea that Candida colonization could spread by contiguity from the oropharynx to the trachea and the tracheostomy site. The proximity of the tracheostomy to the central venous catheter (CVC) insertion site could be considered a predisposing factor for the development of candidemia. Therefore, we suggested topical nystatin prophylaxis in order to reduce the incidence of candidemia. From an operative point of view, we have encouraged prophylactic interventions consisting of oral nystatin administration (500,000 IU) along with oral and tracheostomy site brushings four times a day. These measures led to a statistically significant reduction of candidemia during the first 7 months of nystatin use: in detail, while 12 of 124 patients (9.7\%) developed at least one laboratory confirmed candidemia during the whole of 2011, no blood cultures were positive for Candida spp. in 59 patients admitted to the ICU during the period from January to July $2012(P=0.01$; Figure 1).

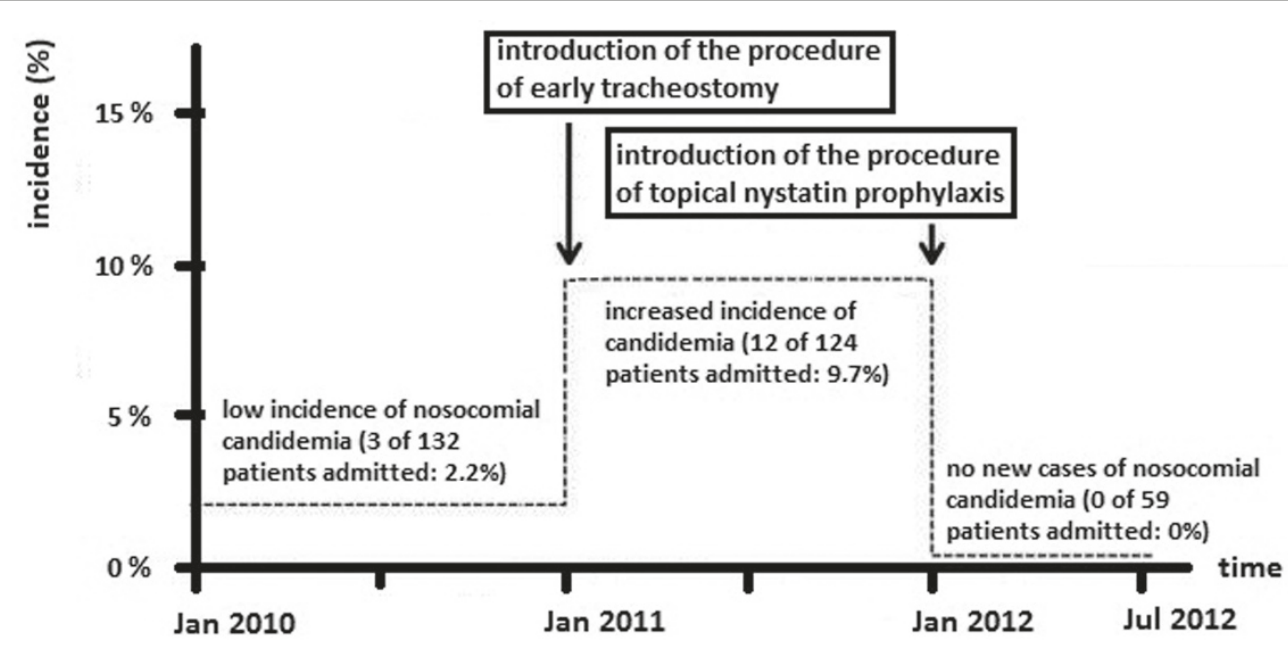

Figure 1. Effects of introduction of topical nystatin prophylaxis. The result of introduction of topical nystatin prophylaxis in neurosurgical ICU patients undergoing early tracheostomy was a dramatic reduction of the incidence of candidemia. 
Our preliminary data add another aspect to this line of research: topical nystatin prophylaxis combined with improved CVC management resulted in a significant decrease in incidence of candidemia. This result is probably allowed by the reduction of the degree of
Candida colonization of the upper respiratory airways in our tracheostomized patients.

Nevertheless, larger clinical trials are needed to understand the real impact of this procedure.

\section{Author's response}

Filomena Puntillo and Mariateresa Giglio

The letter of Ceccarelli and colleagues underlines an important issue in the debate about the prevention of catheter-related bloodstream infections in ICU patients. We agree that the closeness of the tracheostomy to the CVC insertion site could be considered a predisposing factor for the development of candidemia and that by reducing the degree of Candida colonization of the upper respiratory airways in tracheostomized patients, the incidence of candidemia can be decreased. These preliminary data support the idea that what we really need, in order to reduce the incidence of candidemia episodes, are simple, cheap and protocolized strategies. Topical nystatin prophylaxis and a careful management of CVCs are all examples of this kind of intervention. Larger trials are warranted to confirm this hypothesis.

\section{Abbreviations}

CVC, central venous catheter.

\section{Competing interests}

This study was sponsored by Pfizer.

\section{Author details}

'Department of Public Health and Infectious Diseases, University of Rome "Sapienza", Italy. ${ }^{2}$ Department of Emergency Medicine, University of Rome

"Sapienza", Italy.

Published: 18 February 2013

\section{References}

1. Barrett AP: Evaluation of nystatin in prevention and elimination of oropharyngeal Candida in immunosuppressed patients. Oral Surg Oral Med Oral Pathol 1984, 58:148-151.
2. Prentice AG: Oral and gastrointestinal candidosis: prophylaxis during immunosuppressive therapy. Mycoses 1989, 32(Suppl 2):42-46.

3. Desai MH, Rutan RL, Heggers JP, Herndon DN: Candida infection with and without nystatin prophylaxis. A 11-year experience with patients with burn injury. Arch Surg 1992, 127:159-162.

4. Austin N, Darlow BA, McGuire W: Prophylactic oral/topical non-absorbed antifungal agents to prevent invasive fungal infection in very low birth weight infants. Cochrane Database Syst Rev 2009, 4:CD003478.

5. Giglio M, Caggiano G, Dalfino L, Brienza N, Alicino I, Sgobio A, Favale A, Corett C, Montagna MT, Bruno F, Puntillo F: Oral nystatin prophylaxis in surgical/ trauma ICU patients: a randomised clinical trial. Crit Care 2012, 16:R57.

doi:10.1186/cc11921

Cite this article as: Ceccarelli $\mathrm{G}$, et al: Topical nystatin prophylaxis: a likely viable measure for reducing impact of candidemia in neurosurgical patients with early tracheostomy intubation. Critical Care 2013, 17:414. 